\title{
Níveis Diários de Arraçoamento para Alevinos de Tilápia do nilo (Oreochromis Niloticus, L.) Cultivados em Baixas Temperaturas
}

\section{Daily Ration Levels for Nile Tilapia (Oreochromis Niloticus, $L$.) Fingerlings Cultivated in Low Temperatures}

\author{
Nandeyara Ribeiro Marques ${ }^{1}$, Carmino Hayashi², \\ Claudemir Martins Soares ${ }^{3}$, Telma Soares ${ }^{4}$
}

\begin{abstract}
Resumo
O experimento foi realizado no Laboratório de Aqüicultura da Universidade Estadual de Maringá, com o objetivo de avaliar o nível ideal de arraçoamento para alevinos de tilápia do Nilo (Oreochromis niloticus). Foram utilizados oitenta alevinos com peso médio inicial de $0,88 \pm 0,12 \mathrm{~g}$ e comprimento inicial médio de $3,76 \pm 0,20 \mathrm{~cm}$, distribuídos em 16 tanques-rede situados em quatro caixas de $1000 \mathrm{~L}$. Cada tanque-rede com cinco peixes foi considerado como unidade experimental. O delineamento foi inteiramente casualizado, com quatro tratamentos e quatro repetições (4, 7, 10 e $13 \%$ do peso vivo). Os animais foram alimentados com ração contendo $30 \%$ de proteína bruta. As variáveis avaliadas foram peso final médio (PF), ganho de peso (GP), conversão alimentar (CA), comprimento final médio (CF) uniformidade do lote (UNI) e sobrevivência (S). O PF, CF e CA apresentaram relação linear positiva $(\mathrm{P}<0,05)$. O GP apresentou relação quadrática $(\mathrm{P}<0,04)$, com ponto de máximo em 11,55\%. A UNI e a $\mathrm{S}$ não apresentaram diferenças significativas $(\mathrm{P}>0,05)$ entre os tratamentos. Os parâmetros físico-químicos permaneceram dentro da faixa recomendada para os peixes. Concluiu-se que o nível ideal de arraçoamento para alevinos de tilápia do Nilo em uma temperatura média de $23^{\circ} \mathrm{C}$ é $11,55 \%$ do peso vivo dos mesmos.
\end{abstract}

Palavras-chave:Alimentação. Arraçoamento. Desempenho. Nutrição. Oreochromis niloticus. Tilápia do Nilo.

\begin{abstract}
The experiment was carried out in the Aquaculture Laboratory from the State University of Maringá, with the purpose of evaluating the Nile tilapia fingerlings ideal ration levels. Eighty fingerlings were used, with initial average weight of $0.88 \pm 0.12 \mathrm{~g}$ and initial average length of $3.76 \pm 0.20 \mathrm{~cm}$, divided in 16 net cages distributed in four water tanks $(1000 \mathrm{~L})$, with five fish each. The design was completely randomized with four treatments and four replications $(4,7,10$ and $13 \%$ of the fish biomass). They were fed with a diet of $30 \%$ gross protein. The evaluated variables were, final average weight (FW), weight gain (WG), food conversion (FC), final average length (FL), size variation weight (SW) and survival (S). The FW, FL and FC presented positive linear relation $(\mathrm{P}<0,05)$. The WG presented quadratic relation $(\mathrm{p}<0,04)$ reaching its highest level at $11.55 \%$. The $\mathrm{SW}$ and $\mathrm{S}$ did not show significant differences $(\mathrm{P}<0,05)$ between the treatments. Physical and chemical parameters remained within the appropriate limits for the fish. It may be concluded that the ideal ration level for fingerlings at an average temperature of $23^{\circ} \mathrm{C}$ is $11.55 \%$ of the fish biomass.
\end{abstract}

Key words: feeding; Nile tilapia; nutrition; Oreochromis niloticus; performance.

\footnotetext{
1 Programa de Pós-Graduação em Ecologia de Ambientes Aquáticos Continentais, Universidade Estadual de Maringá. Av. Colombo, 5790; CEP:87020-900. Laboratório de Aqüicultura, Bloco H-78, sala 03. F: 261-4667 E-mail: nandi.bio@ bol.com.br.

2 Professor Titular do Departamento de Biologia, Laboratório de Aqüicultura, Universidade Estadual de Maringá.

Biólogo do Departamento de Biologia, Laboratório de Aqüicultura, Universidade Estadual de Maringá.

Graduada em Ciências Biológicas, Laboratório de Aqüicultura, Universidade Estadual de Maringá.
} 


\section{Introdução}

Tilápia é a denominação comum de uma grande gama de espécies de peixes ciclídeos, que conforme Popma e Phelps (1998) distribuem-se originalmente do centro-sul da África até o norte da Síria. Cerca de 22 espécies de tilápia são cultivadas no mundo, e delas a tilápia do Nilo (Oreochromis niloticus), é uma das mais criadas comercialmente (EL-SAYED, 1999).

De acordo com Lovshin (1997), a distribuição das tilápias pelo mundo começou com o intuito da criação de peixes para a subsistência em países em desenvolvimento. No final dos anos setenta, a espécie O. niloticus demonstrou um alto potencial para a aquicultura, em vários sistemas de criação (LAZARD; ROGNON, 1997).

No Brasil, a tilápia do Nilo, proveniente da Costa do Marfim (África Ocidental), foi introduzida no Nordeste em 1971 e a partir de então distribuída pelo país. A tilápia do Nilo e algumas tilápias vermelhas híbridas são as espécies mais cultivadas no Brasil. A tilápia do Nilo é cultivada desde a bacia do rio Amazonas até o Rio Grande de Sul. O interesse pelo cultivo desta espécie, no Sul e Sudoeste do País, cresceu rapidamente nos últimos oito anos, pela introdução da tecnologia da reversão sexual e a pesca esportiva, representado pelos pesque-pagues. A tilápia é criada em diversos sistemas, desde a cultura semi-intensiva em tanques que recebem dejetos animais, como em cultivo intensivos em "raceways" e tanques-rede. Acredita-se que, no Brasil, metade da produção anual de peixes cultivados sejam de tilápia (LOVSHIN; CIRYNO, 1998).

Segundo Popma e Phelps (1998) a tilápia é, dentro das espécies de peixes mais cultivadas, a que melhor resiste à alta temperatura, à baixa concentração de oxigênio dissolvido e à alta concentração de amônia na água. Já Lahav e Ra'nam (1997) citam que a principal vantagem da tilápia do Nilo é o seu baixo custo relativo, principalmente quanto ao alevino, alimentação e a qualidade da sua carne. A espécie de tilápia preferida para o cultivo é a $O$. niloticus devido ao seu rápido crescimento (LOVSHIN, 1997).

O conhecimento básico da biologia e do manejo dos peixes é importante para os estudos que relacionam nutrição. $\mathrm{Na}$ aqüicultura intensiva, mais de 50\% dos custos de produção são com rações (ElSAYED, 1999). Por esse motivo, tornam-se necessário estudos sobre a quantidade (porcentagem do peso vivo) de ração que deve ser fornecida aos peixes, a fim de se evitar perdas e aumento nos custos com a produção. O nível de arraçoamento a ser fornecido a uma dada espécie varia, principalmente, em função da temperatura da água e da idade do peixe, e o nível de oxigênio dissolvido e de amônia também vão influenciar na quantidade de ração consumida (SANTIAGO; ALDABA; REYES, 1987; KUBITZA, 1997).

Para Castagnolli (1979), o excesso de alimento além de provocar alterações metabólico-digestivas, implica a deterioração da qualidade da água. Existem muitos fatores que influenciam o índice de ingestão alimentar e, conseqüentemente, as taxas de arraçoamento devem ser ajustadas segundo as condições individuais de cada unidade de cultivo (ELLIOT, 1975).

Otubusin (1987), em trabalho realizado com alevinos de Oreochromis niloticus, em condições experimentais, testou três níveis de arraçoamento (3, 5 e $10 \%$ do peso vivo), visando a avaliar qual o nível ideal para melhor desempenho dos peixes. Ele observou que o nível de $10 \%$ a partir dos resultados obtidos foi considerado o mais eficiente para variáveis como ganho de peso e peso final. Fontaine et al. (1997), em experimento com Perca fluviatilis, utilizaram níveis menores (1,2 e 3\% do peso vivo) e concluíram que os três níveis afetaram significativamente o crescimento dos animais, embora a sobrevivência não tivesse sido afetada.

Em trabalho com Scophthalmus maximus, com o fornecimento de $0,25,0,38$ e $1,0 \%$ do peso vivo dos animais em ração, Saether e Jobling (1999) observaram um incremento no peso para os três 
níveis, porém com uma redução no crescimento. No entanto, Storebakken e Austreng (1987), em trabalho com salmão do Atlântico Salmo salar, utilizaram 12 níveis de arraçoamento ( 0,5 a $3,25 \%$, em intervalos de $0,25 \%$ ) e verificaram melhor desempenho dos peixes no tratamento em que foi fornecido menor porcentagem do peso vivo em ração. No trabalho de Santiago, Aldaba e Reyes (1987), com larvas de tilápia do Nilo, foram fornecidos altos níveis de arraçoamento (15, 30, 45 e $60 \%$ do peso vivo), tendo sido obtido maior biomassa para os níveis intermediários ( 30 e $45 \%$ do peso vivo).

Quinton e Blake (1990), realizando experimento com truta arco-íris Oncorhynchus mykiss utilizando três níveis alimentares ( 3,5 e $7 \%$ do peso vivo), chegou a conclusão que o nível de $3 \%$ foi o mais eficiente sem afetar o desempenho dos animais.

Com o objetivo de avaliar o nível alimentar ideal para juvenis de esturjão branco (Acipenser transmontanus) a uma temperatura de $20{ }^{\circ} \mathrm{C}$, Hung e Lutes (1987) verificaram que, dentre os níveis avaliados ( 0,5 a $4,0 \%$ em intervalos de $0,5 \%)$, o nível de $2,0 \%$ foi o melhor, levando em consideração variáveis como peso final e conversão alimentar.

O objetivo do presente experimento foi avaliar o nível ideal de arraçoamento para alevinos de tilápia do Nilo, com base nos parâmetros desempenho e sobrevivência.

\section{Material e Métodos}

O presente experimento foi realizado no Laboratório de Aqüicultura do Departamento de Biologia da Universidade Estadual de Maringá, durante 30 dias. Foram utilizados 80 alevinos de tilápia do Nilo (Oreochromis niloticus) com peso inicial de $0,82 \pm 0,10 \mathrm{~g}$ e comprimento inicial médio de $3,76 \pm 0,20$ $\mathrm{cm}$. Os alevinos foram distribuídos em 16 tanquesrede de 48 litros cada, situados em quatro tanques com capacidade para 1000 litros, abastecidos com água de torneira, à qual foram adicionados $17 \mathrm{~mL}$ de tiossulfeto de sódio, para inativar o cloro. $\mathrm{O}$ delineamento experimental foi o inteiramente casualizado com quatro tratamentos e quatro repetições, sendo cada tanque-rede com cinco alevinos considerado como unidade experimental. Os tratamentos constituíram na variação do cálculo da quantidade do arraçoamento dos alevinos de tilápia do Nilo, sendo essa quantidade correspondente a 4 , 7,10 e $13 \%$ do peso vivo dos mesmos. A quantidade de ração foi verificada a cada dez dias, com a pesagem de cada unidade experimental.

Para a alimentação, foi utilizado o manejo alimentar com o uso de uma ração contendo $30 \%$ de proteína bruta e $3000 \mathrm{Kcal}$ de energia digestível (Tabela 1), formulada de acordo com as exigências do NATIONAL RESEARCH COUNCIL (1993) para tilápia do Nilo. Para a preparação das rações, os ingredientes foram moídos individualmente em moinho tipo faca com peneira $0,5 \mathrm{~mm}$, posteriormente pesados, misturados e umedecidos em água a apenas $50{ }^{\circ} \mathrm{C}$ ( e não mais do que isso), para evitar a desnaturação das proteínas. A ração foi peletizada em moinho de carne e seca em estufa de ventilação forçada a $55^{\circ} \mathrm{C}$, e, posteriormente, desintegrada e peneirada para que fosse fornecida em tamanho adequado a idade dos peixes. $\mathrm{O}$ arraçoamento foi realizado quatro vezes ao dia $(8: 00,11: 00,14: 00 \mathrm{e}$ 17:00) e, no período da manhã, as sobras de ração eram retiradas dos tanque-rede por meio de sifonagem. 
Tabela 1 - Composição em ingredientes e bromatológicas da ração fornecida aos alevinos de tilápia do Nilo

\begin{tabular}{llll}
\hline Ingredientes & $(\%)$ & \multicolumn{2}{c}{ Composição } \\
\hline Farelo de soja $^{\text {a }}$ & 49,92 & Proteína bruta (\%) & 30,0 \\
Milho moído $^{\mathrm{a}}$ & 35,96 & Energia digestível (kcal/kg) & 3000 \\
Proteinoso de milho $^{\mathrm{a}}$ & 9,06 & Amido (\%) & 29,03 \\
Óleo de soja $^{\mathrm{a}}$ & 0,63 & Gordura (\%) & 4,00 \\
Fosfato bicálcico $^{\mathrm{b}}$ & 2,41 & Fibra bruta $(\%)$ & 3,22 \\
Calcáreo $^{\mathrm{b}}$ & 0,89 & Lisina (\%) & 1,60 \\
Sal & 0,50 & Metionina + Cistina (\%) & 0,90 \\
Premix vit-mineral $^{\mathrm{c}}$ & 0,50 & Fósforo Total $(\%)$ & 0,80 \\
DL-metionina 99 & 0,10 & Cálcio (\%) & 1,00 \\
BHT & 0,02 & Linoléico $(\%)$ & 1,51 \\
\hline
\end{tabular}

${ }^{\mathrm{a}}$ De acordo com os dados de análise bromatológica e digestibilidade de Meurer, Hayashi e Boscolo (2000), ${ }^{\mathrm{b}}$ de acordo com os dados de Rostagno, Silva e Costa, (1994). ${ }^{\mathrm{c}}$ Níveis de garantia por kg do produto: Vit. A - 2.000.000 UI; Vit. D3: 30.000; Vit. E: 5.000 mg; Vit. K 300,56 mg; Vit. $B_{1}: 1.199 .52$ mg; Vit $B_{2}: 2.000$ mg; Vit $B_{6}: 1.000,316$ mg; Vit. B 12 mg; Vit. C: 15.999,48; Ácido fólico: 199,9984 mg; Ácido nicotínico: 10.000 mg; Pantetonato de cálcio: 6.550,2 mg; Biotina: 50 mg; Cloreto de colina: 339.996 mg; Ferro: 4.000,2 mg; Cobre: 500 mg; Manganês: 4.998 .8 mg; Cobalto: 101,1012 mg; Zinco: 3.599,36 mg; Iodo: 199,64 mg; Antioxidante: $20.000 \mathrm{mg}$.

Os parâmetros físico-químicos da água $(\mathrm{pH}$, condutividade elétrica e oxigênio dissolvido) foram monitorados semanalmente as 9:00 horas e a temperatura duas vezes ao dia (manhã e tarde), durante todo o período experimental.

As variáveis avaliadas foram peso médio final, comprimento médio final, ganho de peso, conversão alimentar, uniformidade em peso do lote e sobrevivência. Para o cálculo do ganho de peso e da conversão alimentar foram utilizadas as fórmulas: $\mathrm{GP}=($ Peso médio final - Peso médio inicial $)$ e $\mathrm{CA}=$ (Ração consumida / ganho de peso). O fator de condição foi calculado com base na equação: $\mathrm{FC}=$ $\left(\right.$ Peso/Comp $\left.{ }^{3}\right)$ x 100. Para avaliar a uniformidade dos lotes de peixes em cada, tanque realizou-se a adaptação de uma equação proposta por Furuya et al. (1998), para determinar a uniformidade em peso de peixes. Utilizando-se as medidas de peso total dos peixes com base nos dados de cada unidade experimental, calculou-se a média, sendo então quantificados o número de indivíduos que se apresentavam com o peso dentro do intervalo correspondente a $20 \%$ acima e abaixo da média de cada unidade experimental.

$$
\mathrm{U}=\frac{\mathrm{N}_{ \pm 20 \%}}{\mathrm{Nt}} \times 100
$$

onde:

$\mathrm{U}=$ Uniformidade do lote: variação no peso total $(\%)$;

$\mathrm{N}_{\mathrm{t}}=$ número total de peixes em cada unidade experimental;

$\mathrm{N}_{ \pm 20}=\mathrm{n}^{\circ}$ de animais com peso total $\pm 20 \%$ em torno da média da unidade experimental

Ao final do experimento, os peixes de cada unidade experimental foram pesados em balança analítica e medidos individualmente para avaliação das variáveis. De posse desses dados, eles foram submetidos à análise de variância ao nível de 5\% de probabilidade e, em caso de diferenças estatísticas, foi aplicado análise de regressão polinomial, ambos pelo programa computacional SAEG-Sistema de Análises Estatísticas e Genéticas (UNIVERSIDADE FEDERAL DE VIÇOSA, 1983). 


\section{Resultados e discussão}

Os resultados médios das variáveis físico-químicas da água foram $22,0 \pm 2,10^{\circ} \mathrm{C}, 24,0 \pm 2,18^{\circ} \mathrm{C}, 6.43 \pm$ $0,48 \mathrm{mg} / \mathrm{L}, 7,41 \pm 0,41$ e $0,92 \pm 0,041 \mathrm{mS} / \mathrm{cm}$, respectivamente para temperatura matutina, temperatura vespertina, oxigênio dissolvido, $\mathrm{pH}$ e condutividade elétrica. Não houve diferenças estatísticas da qualidade da água e temperatura entre os tratamentos, e, de acordo com Egna e Boyd (1997), estes valores estão dentro dos recomendados para a piscicultura.

Os valores médios de desempenho produtivo dos alevinos de tilápia do Nilo $O$. niloticus, submetidos aos diferentes níveis de arraçoamento estão apresentados na Tabela 2. peso no menor nível fornecido. Otubusin (1987) relata que o nível de $10 \%$ foi o mais eficiente para alevinos de tilápia do Nilo, estando este valor próximo ao do obtido neste trabalho.

A conversão alimentar dos alevinos apresentou uma relação linear positiva $(\mathrm{P}<0,05)$ em função do aumento dos níveis alimentares testados. Esse resultado demonstra que houve redução no aproveitamento da ração com o aumento do nível de arraçoamento, fato este confirmado pelas sobras de ração no tratamento onde foi testado o maior nível. Discordando deste trabalho, Fontaine et al.(1997), utilizando alevinos de Perca fluvialis, observaram uma melhora na conversão alimentar à medida que os níveis de arraçoamento foram aumentados.

Tabela 2 - Valores médios de desempenho produtivo dos alevinos de tilápia do Nilo em função dos diferentes níveis de arraçoamento.

\begin{tabular}{lrrrrr}
\hline & \multicolumn{3}{c}{ Níveis de Arraçoamento (\% peso vivo) } & \\
\cline { 2 - 5 } PARÂMETROS & 4 & 7 & 10 & 13 & CV \\
\hline Peso médio Inicial & 0,88 & 0,88 & 0,89 & 0,87 & 1,59 \\
Peso médio Final $^{1}$ & 2,77 & 3,54 & 4,97 & 4,72 & 14,22 \\
Ganho médio de peso $^{2}$ & 1,88 & 2,66 & 4,09 & 3,85 & 18,15 \\
Conversão alimentar $^{3}$ & 1,07 & 1,41 & 1,28 & 1,93 & 19,74 \\
Comprimento médio final $^{4}$ & 5,43 & 5,78 & 6,54 & 6,35 & 10,48 \\
Fator de Condição $^{\mathrm{ns}}$ & 7,13 & 7,99 & 8,86 & 8,21 & 15,80 \\
${\text { Uniformidade do lote }(\%)^{\mathrm{ns}}}$ & 83,75 & 71,50 & 60,00 & 7,50 & 20,69 \\
${\text { Sobrevivência }(\%)^{\mathrm{ns}}}$ & 90,00 & 90,00 & 100,00 & 90,00 & 16,44 \\
\hline
\end{tabular}

${ }^{1}$ Efeito linear $(\mathrm{p}<0,05) \mathrm{Y}=1,9366+0,2430 \mathrm{X}, \mathrm{r}^{2}=0,83 ;{ }^{2}$ Efeito quadrático $(\mathrm{p}<0,04) \mathrm{Y}=-9,9947+4,8850 \mathrm{X}-0,2115 \mathrm{X} 2, \mathrm{r}^{2}$ $=0,84 ;{ }^{3}$ Efeito linear $(\mathrm{p}<0,05) \mathrm{Y}=51,1445+0,3570 \mathrm{X}, \mathrm{r}^{2}=0,78 ;{ }^{4}$ Efeito linear $(\mathrm{p}<0,05) \mathrm{Y}=0,7314+0,0811 \mathrm{X}, \mathrm{r}^{2}=0,74 ;{ }^{\mathrm{ns}}$ não houve diferenças estatísticas $(\mathrm{p}>0,05)$.

O ganho de peso apresentou uma relação quadrática $(\mathrm{p}<0,04)$ com o aumento do nível de arraçoamento com ponto máximo de $11,55 \% \mathrm{em}$ percentagem do peso vivo em ração para alevinos de tilápia do Nilo. Santiago, Aldaba e Reyes (1987) trabalharam com larvas de tilápia do Nilo e concluíram que níveis de 30 a $40 \%$ de ração são os mais indicados para o desempenho dos animais. Storebakken e Austreng (1987), realizaram experimento com alevinos de salmão do Atlântico "Salmo salar" utilizando os níveis 0,5 a 3,25\% em intervalos de $0,25 \%$, e observaram que houve um menor ganho de
Santiago, Aldaba e Reyes (1987) observou, para larvas de tilápia do Nilo, que a conversão alimentar foi mais eficiente quando era fornecido 30 a $40 \%$ do peso vivo dos animais em ração. Storebakken e Austreng (1987), testando os níveis de 0,5 a 3,25\% em intervalos de $0,25 \%$, observaram que a conversão alimentar foi mais eficiente nos menores níveis. Tabata et al. (1988), em trabalho realizado com truta arcoíris (Salmo irideus) com níveis de arraçoamento de $2,3,4,5$ e $6 \%$ do peso vivo, concluíram que a conversão foi mais eficiente no nível de 3\%, resultados que diferem do obtido neste experimento. 
Os valores de peso e comprimento médios finais apresentaram uma relação linear positiva $(\mathrm{p}<0,05)$. Resultados similares foram obtidos por Otubusin (1987), com alevinos de tilápia do Nilo, e por Storebakken e Austreng (1987),como salmão do atlântico Salmo salar, e em ambos os casos verificouse que níveis acima de $1 \%$ do peso vivo não afetaram de forma significativa o peso e o comprimento dos animais. Os resultados obtidos por Fontaine et al.(1997) descrevem que o crescimento dos alevinos de Perca fluvialis foi afetado pelo aumento do nível de arraçoamento, tendo adquirido um maior comprimento médio final os animais submetidos aos menores níveis. Diferindo desse trabalho, Jobling e Baardivik (1994) observou, para Salvelinus alpinus, que menores taxas de arraçoamento promovem maior crescimento.

Com relação ao fator de condição, este parâmetro não apresentou diferenças estatísticas $(p>0,05)$. Discordando deste resultado, Hung e Lutes (1987), trabalhando com o esturjão branco (Acipenser transmontanus), observaram que o fator de condição apresentou diferenças estatísticas significativas $(\mathrm{p}<0,05)$. No entanto, Quinton e Blake (1990) verificaram que, para a truta arco-íris (Oncorhynchus mykiss), o fator de condição não apresentou diferenças estatísticas significativas ( $>>0,05$ ). Os trabalhos de Storenbakken e Austreng (1987) e de Santiago, Aldaba e Reyes (1987) não relataram sobre o fator de condição. Tabata et al. (1998), ao estudarem a truta arco-íris (Salmo iridens) observaram que os valores do fator de condição apresentaram relação linear positiva $(\mathrm{p}<0,05)$ com o aumento dos níveis de arraçoamento.

As taxas de sobrevivência e uniformidade em peso dos alevinos não apresentaram diferenças estatísticas ( $>0,05$ ). Resultado semelhante foi encontrado por Saether e Jobling (1999), que trabalharam com Scophthalmus maximus e não observaram mortalidade. Ao contrário, Tabata et al. (1998) observaram que as taxas de mortalidade foram crescentes com o aumento dos níveis de arraçoamento. A sobrevivência não foi afetada pelos tratamentos no trabalho de Storenbakken e Austreng (1987), dado que foi confirmado por este estudo, com a diferença na porcentagem obtida (81\%). A uniformidade em peso dos animais, reflete a variação entre os indivíduos e o fornecimento de maiores porcentagens do peso vivo em ração deve reduzir a concorrência entre os indivíduos. Isso acarreta uma menor variação entre eles e, conseqüientemente, uma maior uniformidade. No entanto, nos níveis utilizados, esse efeito não apresentou diferenças significativas $(p>0,05)$.

O efeito quadrático do parâmetro relacionado ao peso dos alevinos pode ser explicado devido ao fato de os menores níveis não possibilitarem uma ingestão do nível adequado de nutrientes para o bom crescimento dos animais. O nível de arraçoamento acima dos $12 \%$ levou a uma diminuição do ganho de peso. Neste nível havia sobra de ração no fundo das hapas e essas sobras devem ter perdido parte de seus nutrientes (principalmente vitaminas e minerais), devido à lixiviação destes para a água. Esses nutrientes podem ter sido ingeridos em algum momento pelos peixes deste tratamento e eles, quando eram arraçoados novamente, não tinham apetite para se alimentar com a ração nova (não lixiviada).

\section{Conclusão}

Conclui-se que o nível ideal de arraçoamento para alevinos de tilápia do Nilo (O. niloticus), a uma temperatura média da água de $23{ }^{\circ} \mathrm{C}$ com melhor desempenho produtivo, é de $11,55 \%$ do peso vivo dos mesmos.

\section{Referências}

CASTAGNOLLI, N. Tecnologia da alimentação de peixes. In: _____. Fundamentos de nutrição de peixes. São Paulo: Livroceres, 1979. 105p.

EGNA, H. S.; BOYD, C. E. Dynamics of pond aquaculture. Boca Raton: CRC Press, 1997. 342p.

EL-SAYED, A. F. M. Alternative dietary protein sources for farmed tilapia, Oreochromis spp. Aquaculture, Amsterdam, v.179, n.2, p.149-168, 1999. 
ELLIOT, J. M. Number of meals in a day, maximum weight of food consumed in a day and maximum rate of feeding for brown trout, Salvelinus fontinalis L. Freshwater biology, Oxford, v.5, n.2, p.287-303, 1975.

FONTAINE, P.; GARDEUR, J. N., KESTEMONT, P.; GEORGES, A. Influence of feeding level on growth, intraspecific weight variability and sexual growth dimorfhism of Eurasian perch Perca fluvialis L. reared in a recirculation system. Aquaculture, Amsterdam, v.157, n.2, p.1-8, 1997.

FURUYA, W. M. et al. Dietas peletizada e extrusada para machos revertidos de tilápia do Nilo (Oreochromis niloticus) na fase de terminação. Ciência Rural, Santa Maria, v.28, n.3, p.483-487, 1998.

HUNG, S. S. O.; LUTES, P. B. Optimum feeding rate of hatchery-produced juvenile white sturgeon Acipenser transmontanus: at $20^{\circ} \mathrm{C}$. Aquaculture, Amsterdam, v.65, n.2, p.307-317, 1987.

JOBLING, M.; BAARDIVIK, B. M. The influence of environmental manipulations on inter and intra - individual varation in food acquisition and growth performance of Artic charr Salvelinus alpinus. Journal of Fish Biology, London, v.41, n.5, p.1067-1087, 1994.

KUBITZA, F. Qualidade do alimento, qualidade da água e manejo alimentar na produção de peixes. In: SIMPÓSIO SOBRE MANEJO E NUTRIÇÃO DE PEIXES, 1., 1997, Piracicaba. Anais... Piracicaba: CBNA, 1997. p.63-116.

LAHAV, E.; RA'NAN, Z. Salinity tolerance of genetically produced tilapia (Oreochromis) hybrids. Israeli Journal of Aquaculture-Bamidgeh, Bamidgeh, v.49, n.3, p.160$165,1997$.

LAZARD, J; ROGNON, X. Genetic diversity of tilapia and aquaculture development in Côte D'Ivoire and Niger. Israeli Journal of Aquaculture-Bamidgeh, Bamidgeh, v.49, n.2, p.90-98, 1997.

LOVSHIN, L. L.; CYRINO, J. E. P. Status of commercial fresh water fish culture in Brazil. In: SIMPÓSIO SOBRE MANEJO E NUTRIÇÃO DE PEIXES, 2., 1998, Piracicaba. Anais... Piracicaba: CBNA, 1998. p.1-20.

LOVSHIN, L. L. Tilapia farming: A Growing Worldwide Aquaculture Industry. In: SIMPÓSIO SOBRE MANEJO E NUTRIÇÃO DE PEIXES, 1, Piracicaba, 1997. Anais... Piracicaba: CBNA, 1997.p.137-164.

MEURER, F.; HAYASHI, C.; BOSCOLO, W. R. Digestibilidade aparente da proteína bruta, matéria seca e energia bruta de alguns alimentos protéicos para tilápia do Nilo (Oreochromis niloticus, L). In: SIMPÓSIO BRASILEIRO DE AQÜICULTURA, 11., Florianópolis, 2000. Anais... Florianópolis: SIMBRAq, 2000. CD-Rom.

NATIONAL RESEARCH COUNCIL. Nutrient requirements of fish. Washington, D.C. : National Academy, 1993.

POPMA, T. J.; PHELPS, R. P. Status report to commercial tilápia producers on monosex fingerling productions techniques. In: AQUICULTURA BRASIL, 1998, Recife. Anais... Recife: SIMBRAQ, 1998. p.27-145.

OTUBUSIN, S. O. Effects of different levels of blood meal in pelleted feeds on tilapia, Oreochromis niloticus, production in floating bamboo net-cages. Aquaculture, Amsterdam, v.65, n.3/4, p.263-266, 1987.

QUINTON, J. C.; BLAKE, R. W. The effect of feed cycling and ration level on the compensatory growth response in rainbow trout, Oncorhynchus mykiss. Journal of Fish Biology, London, v.37, n.1, p.33-41, 1990.

ROSTAGNO, H. S.; SILVA, D. J.; COSTA, P. M. A. Composição de alimentos e exigências nutricionais de aves e suínos (Tabelas brasileiras). Viçosa: Imprensa Universitária, 1994.

SAETHER, B. S.; JOBLING, M. The effects of ration level on feed intake and growth and compensatory growth after restricted feeding, in turbot Scophthalmus maximus L. Aquaculture Research, Oxford, v.30, n.9 p.647-652, 1999.

SANTIAGO, C. B.; ALDABA, M. B.; REYES, O. S. Influence of feeding rate and diet form on growth and survival of Nile Tilapia (Oreochromis niloticus) fry. Aquaculture, Amsterdam, v.64, n.4, p.277-282, 1987.

STORENBAKKEN, T.; AUSTRENG, E. Ration level for salmonids: growth, survival, body composition and feed conversion in Atlantic Salmon fry and fingerlings. Aquaculture, Amsterdam, v.60, n.3/4, p.189-203, 1987.

TABATA, Y. A.; RIGOLINO, M. G.; NETO, B. C. S.; PAIVA, P.; ISHIKAWA, C. M. Influência de diferentes taxas de arraçoamento n crescimento de truta arco-íris, Salmo irideus Gibbons. Boletim do Instituto de Pesca, São Paulo, v.15, n.1, p.31-38, 1998.

UNIVERSIDADE FEDERAL DE VIÇOSA. SAEG Sistema para análises estatísticas e genéticas. Versão 7.1. Viçosa, MG., 1983. 150p. Manual do usuário. 
